VOL. $72(2005)$ [161-172]

\title{
IMPLICIT VECTOR EQUILIBRIUM PROBLEMS VIA NONLINEAR SCALARISATION
}

\author{
JUN Li AND NAN-JING HUANG
}

\begin{abstract}
The purpose of this paper is to introduce a nonlinear scalarisation function for solving a class of implicit vector equilibrium problems. We prove a scalarisation lemma to show the relation between the implicit vector equilibrium problem and the nonlinear scalarisation function. Then we derive some new existence theorems for solutions of implicit vector equilibrium problems, using the scalarisation lemma and the FKKM theorem.
\end{abstract}

\section{INTRODUCTION}

Given a nonempty set $A$ and a scalar bifunction $f: A \times A \rightarrow R=(-\infty, \infty)$ with $f(x, x) \geqslant 0$ for all $x \in A$, the scalar equilibrium problem for $f$ is to find $x^{*} \in A$ such that

$$
\text { Equilibrium problem } f\left(x^{*}, y\right) \geqslant 0, \quad \forall y \in A \text {. }
$$

It is well known that the equilibrium problem is closely related to variational inequalities and complementarity problems, and optimisation and control problems as well as problems arising in game theory, mechanics and physics, economics and finance, and operations research, (see, for example, $[1,2,3,4,5,6,7,8,9,10,12,13,14,15,16$, $17,18,19,20,21,22,23]$ and the references therein). Recently, many authors have studied the equilibrium problem in the scalar case (see $[5,6,13,17,19])$. At the same time, this problem has been generalised to the vector case (see $[4,13,17])$, even to the multivalued $[2,20]$ and system [1] cases.

On the other hand, scalarisation plays an important role in the study of vector equilibrium problems and vector variational inequality problems (see, for example, [9, $10,14,15,16,23]$ and the references therein), especially in algorithm design. In 1990, Gerth and Weidner [12] firstly introduced the nonlinear scalarisation method with respect to general domination sets. In 2001, Gong [15] derived the scalarisation results for vector

\section{Received 3rd May, 2005}

The first author was supported by the Youth Foundation of Sichuan Education Department of China, the National Natural Science Foundation of Sichuan Education Department of China (2004C018), the Foundation of Sichuan Science and Technology Department of China and the second author was supported by the National Natural Science Foundation of China.

Copyright Clearance Centre, Inc. Serial-fee code: 0004-9727/05 \$A2.00+0.00. 
equilibrium problems under an assumption of convexity. Recently, Chen and Yang [10] introduced a nonlinear scalarisation function for a variable domination structure, and this nonlinear scalarisation function was then applied to characterise the weakly nondominated solution of multicriteria decision making problems and the solution of vector variational inequalities.

Let $X$ be a real Hausdorff topological vector space and $K$ be a nonempty subset of $X$. Let $C: X \rightarrow 2^{X}$ be a set-valued mapping such that for each $x \in X, C(x)$ is a proper, pointed, closed and convex cone in $X$. Let $\bar{C}=\bigcap_{x \in X} C(x)$ with int $\bar{C} \neq \emptyset$. For a given vector valued bifunction $f: K \times K \rightarrow X$, the non-dominated vector equilibrium problem consists of finding $x^{*} \in K$ such that

(Non-dominated vector equilibrium problem) $f\left(x^{*}, y\right) \notin-\operatorname{int} C\left(x^{*}\right), \quad \forall y \in K$.

The point $x^{*} \in K$ is called a solution of non-dominated vector equilibrium problem, and we denote by $W(f, K)$ the set of all solutions of non-dominated vector equilibrium problem. In [23], Wu gave a scalarisation theorem for non-dominated vector equilibrium problem and established existence of solutions employing the famous theorem of Fan, Knoster, Kuratowski and Marzurkiewica (the FKKM theorem) without any assumption of monotonicity.

Let $X$ and $Y$ be real Hausdorff topological vector spaces, $K$ a nonempty subset of $X$. Let $C: X \rightarrow 2^{Y}$ be a point-to-set mapping such that for any $x \in X, C(x)$ is a proper, pointed, closed and convex cone in $Y$. Let $\bar{C}=\bigcap_{x \in X} C(x)$ with int $\bar{C} \neq \emptyset$.

As is well known, properness means that $C(x) \neq Y$ for any $x \in X$. A nonempty subset $P$ of $Y$ is called a pointed and convex cone in $Y$ if

(i) $P+P=P$;

(ii) $\lambda P \subseteq P$ for all $\lambda>0$ and

(iii) $P \cap\{-P\}=\{0\}$. It is easy to see that $\bar{C}$ is also a proper, closed and convex cone in $Y$.

In this paper, we consider the following implicit vector equilibrium problem. Given a vector valued function $g: K \rightarrow K$ and a vector valued bifunction $f: K \times K \rightarrow Y$, find a point $x^{*} \in K$ such that

(Implicit vector equilibrium problem) $f\left(g\left(x^{*}\right), y\right) \notin-\operatorname{int} C\left(x^{*}\right), \quad \forall y \in K$.

The point $x^{*} \in K$ is called a solution of implicit vector equilibrium problem, and we denote by $S(f, g, K)$ the set of all solutions of the implicit vector equilibrium problem. $\mathrm{Li}$, Huang and Kim [21] have studied the existence of solutions of implicit vector equilibrium problem without monotonicity and convexity. In [18], Huang, $\mathrm{Li}$ and Thompson gave characterisations of $S(f, g, K)$ under the assumptions of non-monotonicity and weakly $C$-pseudomonotonicity, respectively. 
If $g: K \rightarrow K$ is the identity mapping, then the implicit vector equilibrium problem reduces to the vector equilibrium problem, which has been studied by many authors (see, for example, [13] and the references therein). We denote by $S(f, K)$ the set of all solutions of the vector equilibrium problem.

If $X=Y$, then the vector equilibrium problem reduces to the non-dominated vector equilibrium problem non-dominated vector equilibrium problem, which was considered by Wu [23].

The purpose of this paper is to introduce a nonlinear scalarisation function for solving the implicit vector equilibrium problem. We prove a scalarisation lemma showing the relationship between the implicit vector equilibrium problem and the nonlinear scalarisation function and then derive some new existence theorems of solutions for the implicit vector equilibrium problems by using the scalarisation lemma and the FKKM theorem. This paper is organised as follows. In the next section, we recall some definitions and the FKKM theorem, introduce a nonlinear scalarisation function which is a generalisation of [10] or [12], and present some important properties of this nonlinear scalarisation function. In Section 3, we first prove a scalarisation lemma to show the relationship between the implicit vector equilibrium problem and the nonlinear scalarisation function, and then derive some new existence theorems for solutions of the implicit vector equilibrium problems using the scalarisation lemma and the FKKM theorem.

\section{Preliminaries}

We first recall some definitions.

Definition 2.1: Let $X$ and $Y$ be two topological vector spaces, $K$ a nonempty subset of $X$. Let $W: K \rightarrow 2^{Y}$ be a point-to-set mapping. The graph of $W$, denoted by $\operatorname{Graph}(W)$, is

$$
\operatorname{Graph}(W)=\{(x, z) \in K \times Y: x \in K, z \in W(x)\} .
$$

The point-to-set mapping $W$ is said to have closed graph in $K \times Y$ if, for any $\left(u_{n}, x_{n}\right)$ $\in \operatorname{Graph}(W)$ with limits $\left(u^{*}, x^{*}\right)$, we have $\left(u^{*}, x^{*}\right) \in \operatorname{Graph}(W)$.

Definition 2.2: Let $X$ and $Y$ be two topological vector spaces, $K$ a nonempty subset of $X$. A mapping $f: K \rightarrow Y$ is called affine if, for any $x_{1}, x_{2} \in K$, and $t \in R$ with $t x_{1}+(1-t) x_{2} \in K$,

$$
f\left(t x_{1}+(1-t) x_{2}\right)=t f\left(x_{1}\right)+(1-t) f\left(x_{2}\right) .
$$

REMARK 2.1. It is easy to see that $f$ is affine if and only if for any $x_{i} \in K$ and $t_{i} \in[0,1]$ $(i=1, \ldots, n)$ with $\sum_{i=1}^{n} t_{i}=1$ and $\sum_{i=1}^{n} t_{i} x_{i} \in K$,

$$
f\left(\sum_{i=1}^{n} t_{i} x_{i}\right)=\sum_{i=1}^{n} t_{i} f\left(x_{i}\right) .
$$


Definition 2.3: Let $K$ be a nonempty subset of topological vector space $X$. A point-to-set mapping $G: K \rightarrow 2^{X}$ is called a KKM-map if, for every finite subset $\left\{x_{1}, x_{2}, \ldots, x_{n}\right\}$ of $K, \operatorname{co}\left\{x_{1}, x_{2}, \ldots, x_{n}\right\}$ is contained in $\bigcup_{i=1}^{n} G\left(x_{i}\right)$, where co denotes the convex hull.

In [12], Gerth and Weidner derived the nonconvex separation theorems for any arbitrary set and any not necessarily convex set in a topological vector space. We now introduce a nonlinear scalarisation function based on Gerth and Weidner as follows:

DEFINITION 2.4: Let $X$ and $Y$ be real Hausdorff topological vector spaces, $C: X$ $\rightarrow 2^{Y}$ a point-to-set mapping such that for any $x \in X, C(x)$ is a proper, pointed, closed and convex cone in $Y$. Let $\bar{C}=\bigcap_{x \in X} C(x)$ with int $\bar{C} \neq \emptyset$, and $k^{0} \in$ int $\bar{C}$. We define a nonlinear scalarisation function $\xi_{k^{0}}: X \times Y \rightarrow R$ as follows:

$$
\xi_{k^{0}}(x, y) \stackrel{\text { def }}{=} \inf \left\{\lambda \in R: y \in \lambda k^{0}-C(x)\right\}, \quad \forall(x, y) \in X \times Y .
$$

If $X=Y$, then the nonlinear scalarisation function $\xi_{k^{0}}$ is equivalent to the nonlinear scalarisation function introduced by Chen and Yang [10].

The following results are very important properties of the nonlinear scalarisation function $\xi_{k^{0}}$, which were originally established by Gerth and Weidner in [12] for general sets. In [10], a slight modification in the formulation was given by Chen and Yang.

Lemma 2.1. Let $X$ and $Y$ be real Hausdorf topological vector spaces, $C: X \rightarrow 2^{Y}$ a point-to-set mapping such that for any $x \in X, C(x)$ is a proper, pointed, closed and convex cone in $Y$. Let $\bar{C}=\bigcap_{x \in X} C(x)$ with int $\bar{C} \neq \emptyset$, and $k^{0} \in \operatorname{int} \bar{C}$. For each $\lambda \in R$ and $(x, y) \in X \times Y$, we have

(i) $\xi_{k^{0}}(x, y)<\cdot \lambda \Leftrightarrow y \in \lambda k^{0}-\operatorname{int} C(x)$;

(ii) $\xi_{k^{0}}(x, y) \leqslant \lambda \Leftrightarrow y \in \lambda k^{0}-C(x)$;

(iii) $\xi_{k^{0}}(x, y) \geqslant \lambda \Leftrightarrow y \notin \lambda k^{0}-\operatorname{int} C(x)$;

(iv) $\xi_{k^{0}}(x, y)>\lambda \Leftrightarrow y \notin \lambda k^{0}-C(x)$;

(v) $\xi_{k^{0}}(x, y)=\lambda \Leftrightarrow y \in \lambda k^{0}-\partial C(x)$,

where $\partial C(x)$ is topological boundary of $C(x)$.

Proof: The proof is similar to the proof of [12] (or [10]) and so we omit it.

In the next section, we also need the following FKKM theorem.

LEMMA 2.2. [11] Let $K$ be a nonempty subset of a Hausdorff topological vector space $X$. Let $G: K \rightarrow 2^{X}$ be a KKM-map, such that for any $y \in K, G(y)$ is nonempty closed and $G\left(y^{*}\right)$ is contained in a compact set $D \subseteq X$ for some $y^{*} \in K$. Then there exists $x^{*} \in D$ such that $x^{*} \in G(y)$ for all $y \in K$, that is, $\bigcap_{y \in K} G(y) \neq \emptyset$. 


\section{Main Results}

In this section, we prove some new existence theorems for solutions of implicit vector equilibrium problems. First we derive a scalarisation lemma via the nonlinear scalarisation function introduced in Section 2.

Lemma 3.1. (Scalarisation Lemma.) Let $X$ and $Y$ be real Hausdorff topological vector spaces and $K$ be a nonempty subset of $X$. Let $C: X \rightarrow 2^{Y}$ be a point-to-set mapping such that for any $x \in X, C(x)$ is a proper, pointed, closed and convex cone in $Y$, and $\bar{C}=\bigcap_{x \in X} C(x)$ with int $\bar{C} \neq \emptyset$. Then $x^{*} \in S(f, g, K)$ if and only if

$$
0=\sup \left\{\xi_{k^{0}}\left(x^{*}, 0\right): k^{0} \in \operatorname{int} \bar{C}\right\} \leqslant \inf \left\{\xi_{k^{0}}\left(x^{*}, f\left(g\left(x^{*}\right), y\right)\right): k^{0} \in \operatorname{int} \bar{C}\right\}, \quad \forall y \in K
$$

Proof: Necessity. Suppose that $x^{*} \in S(f, g, K)$. Then,

$$
f\left(g\left(x^{*}\right), y\right) \notin-\operatorname{int} C\left(x^{*}\right), \quad \forall y \in K .
$$

By Lemma 2.1 (iii), for each $y \in K$,

$$
\xi_{k^{0}}\left(x^{*}, f\left(g\left(x^{*}\right), y\right)\right) \geqslant 0, \quad \forall k^{0} \in \operatorname{int} \bar{C} .
$$

Thus,

$$
0 \leqslant \inf \left\{\xi_{k^{0}}\left(x^{*}, f\left(g\left(x^{*}\right), y\right)\right): k^{0} \in \operatorname{int} \bar{C}\right\}, \quad \forall y \in K .
$$

Since $\xi_{k^{0}}\left(x^{*}, 0\right)=\inf \left\{\lambda \in R: 0 \in \lambda k^{0}-C\left(x^{*}\right)\right\}$ and $C\left(x^{*}\right)$ is a pointed cone, we know that $\xi_{k^{0}}\left(x^{*}, 0\right)=0$ for each $k^{0} \in \operatorname{int} \bar{C}$ and so

$$
0=\sup \left\{\xi_{k^{0}}\left(x^{*}, 0\right): k^{0} \in \operatorname{int} \bar{C}\right\} \leqslant \inf \left\{\xi_{k^{0}}\left(x^{*}, f\left(g\left(x^{*}\right), y\right)\right): k^{0} \in \operatorname{int} \bar{C}\right\}, \quad \forall y \in K .
$$

Sufficiency. Assume that $x^{*} \in K$ and for any $y \in K$,

$$
0=\sup \left\{\xi_{k^{0}}\left(x^{*}, 0\right): k^{0} \in \operatorname{int} \bar{C}\right\} \leqslant \inf \left\{\xi_{k^{0}}\left(x^{*}, f\left(g\left(x^{*}\right), y\right)\right): k^{0} \in \operatorname{int} \bar{C}\right\} .
$$

It follows that, for each $k^{0} \in \operatorname{int} \bar{C}, \xi_{k^{0}}\left(x^{*}, f\left(g\left(x^{*}\right), y\right)\right) \geqslant 0$. For each $k^{0} \in \operatorname{int} \bar{C}$, Lemma 2.1 (iii) implies that

$$
f\left(g\left(x^{*}\right), y\right) \notin 0 k^{0}-\operatorname{int} C\left(x^{*}\right)=-\operatorname{int} C\left(x^{*}\right), \quad \forall y \in K .
$$

Hence, $x^{*} \in S(f, g, K)$. This completes the proof.

REMARK 3.1. If $g$ is the identity mapping, then Lemma 3.1 establishes necessary and sufficient conditions for $x^{*} \in S(f, K)$ based on the nonlinear scalarisation function. 
LEMMA 3.2. If we define $\varphi: K \rightarrow 2^{K}$ such that

$$
\varphi(x)=\left\{x^{*} \in K: \xi_{k^{0}}\left(x^{*}, f\left(g\left(x^{*}\right), x\right)\right) \geqslant 0, \forall k^{0} \in \operatorname{int} \bar{C}\right\}, \quad \forall x \in K,
$$

then,

$$
\bigcap_{x \in K} \varphi(x)=S(f, g, K)
$$

Proof: Let $x^{*} \in \bigcap_{x \in K} \varphi(x)$. Then $x^{*} \in \varphi(x)$ for all $x \in K$. That is, $x^{*} \in K$ and $\xi_{k^{0}}\left(x^{*}, f\left(g\left(x^{*}\right), x\right)\right) \geqslant 0$ for all $k^{0} \in \operatorname{int} \bar{C}$ and $x \in K$. Hence,

$$
\inf \left\{\xi_{k^{0}}\left(x^{*}, f\left(g\left(x^{*}\right), x\right)\right): k^{0} \in \operatorname{int} \bar{C}\right\} \geqslant 0, \quad \forall x \in K .
$$

Thus, Lemma 3.1 implies that $x^{*} \in S(f, g, K)$.

Conversely, suppose that $x^{*} \in S(f, g, K)$. Then,

$$
f\left(g\left(x^{*}\right), x\right) \notin-\operatorname{int} C\left(x^{*}\right), \quad \forall x \in K .
$$

By Lemma 2.1 (iii), for each $x \in K$,

$$
\xi_{k^{0}}\left(x^{*}, f\left(g\left(x^{*}\right), x\right)\right) \geqslant 0, \quad \forall k^{0} \in \operatorname{int} \bar{C},
$$

that is, $x^{*} \in \varphi(x)$. Hence,

$$
x^{*} \in \bigcap_{x \in K} \varphi(x)
$$

This completes the proof.

REMARK 3.2. If $g$ is the identity mapping, then in view of Remark 3.1, we obtain from Lemma 3.2 a characterisation of $S(f, K)$ based on the nonlinear scalarisation function.

LEMMA 3.3. Let $X$ and $Y$ be real Hausdorff topological vector spaces, $K$ a nonempty closed subset of $X$. Let $C: X \rightarrow 2^{Y}$ be a point-to-set mapping such that for any $x \in X, C(x)$ is a proper, pointed, closed and convex cone in $Y$, and $\bar{C}=\bigcap_{x \in X} C(x)$ with int $\bar{C} \neq \emptyset$. Let the following assumptions hold:

(i) $g$ and $x \mapsto f(x, \cdot)$ are continuous;

(ii) the point-to-set mapping $W: K \rightarrow 2^{Y}$ has closed graph in $K \times Y$, where $W(x)=Y \backslash(-\operatorname{int} C(x)), \forall x \in K$.

Then for any given $x \in K, \varphi(x)$ is closed or empty, where

$$
\varphi(x)=\left\{x^{*} \in K: \xi_{k^{0}}\left(x^{*}, f\left(g\left(x^{*}\right), x\right)\right) \geqslant 0, \forall k^{0} \in \operatorname{int} \bar{C}\right\} .
$$


Proof: For any given $x \in K$, if $\varphi(x) \neq \emptyset$, then let $\left\{u_{n}\right\} \subset \varphi(x)$ such that $u_{n} \rightarrow u^{*}$. Then $u_{n} \in K$ and

$$
\xi_{k^{0}}\left(u_{n}, f\left(g\left(u_{n}\right), x\right)\right) \geqslant 0, \quad \forall k^{0} \in \operatorname{int} \bar{C} .
$$

Since $K$ is closed, we know that $u^{*} \in K$. By Lemma 2.1 (iii), it follows that

$$
f\left(g\left(u_{n}\right), x\right) \notin-\operatorname{int} C\left(u_{n}\right),
$$

that is,

$$
\left(u_{n}, f\left(g\left(u_{n}\right), x\right)\right) \in \operatorname{Graph}(W) .
$$

From assumption (i), we have $\left(u_{n}, f\left(g\left(u_{n}\right), x\right)\right) \rightarrow\left(u^{*}, f\left(g\left(u^{*}\right), x\right)\right)$. Since $W(x)=Y \backslash(-\operatorname{int} C(x))$ for all $x \in K$ and $W$ has closed graph in $K \times Y$, we obtain

$$
\left(u^{*}, f\left(g\left(u^{*}\right), x\right)\right) \in \operatorname{Graph}(W)
$$

that is,

$$
f\left(g\left(u^{*}\right), x\right) \notin-\operatorname{int} C\left(u^{*}\right) .
$$

Again by Lemma 2.1 (iii), we obtain

$$
\xi_{k^{0}}\left(u^{*}, f\left(g\left(u^{*}\right), x\right)\right) \geqslant 0, \quad \forall k^{0} \in \operatorname{int} \bar{C},
$$

and hence $u^{*} \in \varphi(x)$. Thus $\varphi(x)$ is closed for any $x \in K$. This completes the proof.

Let $g$ be the identity mapping in the proof of Lemma 3.3. From Remark 3.2, we have the following conclusion.

Corollary 3.1. Let $X$ and $Y$ be real Hausdorff topological vector spaces, $K$ a nonempty closed subset of $X$. Let $C: X \rightarrow 2^{Y}$ be a point-to-set mapping such that for any $x \in X, C(x)$ is a proper, pointed, closed and convex cone in $Y$, and $\bar{C}=\bigcap_{x \in X} C(x)$ with int $\bar{C} \neq \emptyset$. Let the following assumptions hold:

(i) $x \mapsto f(x, \cdot)$ is continuous;

(ii) the point-to-set mapping $W: K \rightarrow 2^{Y}$ has closed graph in $K \times Y$, where $W(x)=Y \backslash(-\operatorname{int} C(x)), \forall x \in K$.

Then for any given $x \in K, \varphi(x)$ is closed, possibly empty, where

$$
\varphi(x)=\left\{x^{*} \in K: \xi_{k^{0}}\left(x^{*}, f\left(x^{*}, x\right)\right) \geqslant 0, \forall k^{0} \in \operatorname{int} \bar{C}\right\} .
$$

Now from Lemma 3.3 , we have

$$
\bigcap_{x \in K} \varphi(x)=\bigcap_{x \in K} \overline{\varphi(x)}
$$


where $\overline{\varphi(x)}$ denotes the closure of $\varphi(x)$. It follows from Lemma 3.2 that

$$
\bigcap_{x \in K} \overline{\varphi(x)}=\bigcap_{x \in K} \varphi(x)=S(f, g, K)
$$

and so $S(f, g, K)$ is closed or empty. Thus, in order to prove $S(f, g, K) \neq \emptyset$, we only need to show $\bigcap_{x \in K} \overline{\varphi(x)} \neq \emptyset$.

TheOREM 3.1. Let $X$ and $Y$ be real Hausdorff topological vector spaces, $K$ a nonempty closed convex subset of $X$. Let $C: X \rightarrow 2^{Y}$ be a point-to-set mapping such that for any $x \in X, C(x)$ is a proper, pointed, closed and convex cone in $Y$. Suppose that $\bar{C}=\bigcap_{x \in X} C(x)$ with int $\bar{C} \neq \emptyset$. Let the following assumptions hold:

(i) $f(g(x), x) \notin-\operatorname{int} C(x), \quad \forall x \in K$;

(ii) $y \mapsto f(\cdot, y)$ is affine;

(iii) $g$ and $x \mapsto f(x, \cdot)$ are continuous;

(iv) the point-to-set mapping $W: K \rightarrow 2^{Y}$ has closed graph in $K \times Y$, where $W(x)=Y \backslash(-\operatorname{int} C(x)), \forall x \in K$;

(v) there exist a compact subset $D \subset K$, such that $\exists y_{0} \in K, \forall x \in K \backslash D$ such that

$$
f\left(g(x), y_{0}\right) \in-\operatorname{int} C(x)
$$

Then

$$
\bigcap_{x \in K} \overline{\varphi(x)} \neq \emptyset
$$

that is, $S(f, g, K) \neq \emptyset$, where

$$
\varphi(x)=\left\{x^{*} \in K: \xi_{k^{0}}\left(x^{*}, f\left(g\left(x^{*}\right), x\right)\right) \geqslant 0, \forall k^{0} \in \operatorname{int} \bar{C}\right\} .
$$

Furthermore, $S(f, g, K)$ is compact.

Proof: Since $f(g(x), x) \notin-\operatorname{int} C(x)$ for any $x \in K$, Lemma 2.1 (iii) implies that for any $x \in K, \xi_{k^{0}}(x, f(g(x), x)) \geqslant 0, \forall k^{0} \in \operatorname{int} \bar{C}$. That is, for any $x \in K, x \in \varphi(x)$ and so $\varphi(x)$ is nonempty. By assumptions (iii) and (iv), Lemma 3.3 implies that $\varphi(x)$ is closed. We next show that $\varphi$ is a KKM-map. Suppose that there exist a finite subset $\left\{u_{1}, u_{2}, \ldots, u_{n}\right\}$ of $K$ and $\lambda_{i} \geqslant 0$ with $\sum_{i=1}^{n} \lambda_{i}=1$, such that

$$
\bar{u}=\sum_{i=1}^{n} \lambda_{i} u_{i} \notin \bigcup_{j=1}^{n} \varphi\left(u_{j}\right) .
$$

Then $\bar{u} \notin \varphi\left(u_{j}\right), j=1,2, \ldots, n$. By the definition of $\varphi$, there exist $k_{1}, k_{2}, \ldots, k_{n} \in \operatorname{int} \bar{C}$ such that

$$
\xi_{k_{j}}\left(\bar{u}, f\left(g(\bar{u}), u_{j}\right)\right)<0, \quad j=1,2, \ldots, n
$$


By Lemma 2.1 (i), we have

$$
f\left(g(\bar{u}), u_{j}\right) \in-\operatorname{int} C(\bar{u}), \quad j=1,2, \ldots, n .
$$

Since $K$ is convex, $C(\bar{u})$ is a convex cone and $y \mapsto f(\cdot, y)$ is affine, then

$$
f(g(\bar{u}), \bar{u})=\sum_{j=1}^{n} \lambda_{j} f\left(g(\bar{u}), u_{j}\right) \in-\operatorname{int} C(\bar{u}),
$$

which is a contradiction. So $\varphi$ is a KKM-map. By the assumption (v), there exist a compact subset $D \subset K$, such that $\exists y_{0} \in K, \forall x \in K \backslash D$ such that

$$
f\left(g(x), y_{0}\right) \in-\operatorname{int} C(x) .
$$

Again by Lemma 2.1 (i), we can deduce that for any $x \in K \backslash D$,

$$
\xi_{k^{0}}\left(x, f\left(g(x), y_{0}\right)\right)<0, \quad \forall k^{0} \in \operatorname{int} \bar{C} .
$$

Hence for any $x \in K \backslash D, x \notin \varphi\left(y_{0}\right)$. Then, one has $\varphi\left(y_{0}\right) \subseteq D$. Since $D$ is compact and $\varphi(x)$ is closed for any $x \in K$, we know that $\varphi\left(y_{0}\right)$ is closed and compact. It follows from Lemma 2.2 that $\bigcap_{x \in K} \overline{\varphi(x)} \neq \emptyset$ and so $S(f, g, K) \neq \emptyset$.

From assumption (v), any element outside the set $D$ cannot be a solution of implicit vector equilibrium problem. Therefore, $S(f, g, K)$ must be contained in $D$. Since $S(f, g, K)$ is closed, $D$ is compact and we know that $S(f, g, K)$ is compact. This completes the proof.

EXAMPle 3.1. Let $X=Y=R^{2}, K=[0,1] \times[0,1]$ and $C(x)=R_{+}^{2}=[0, \infty) \times[0, \infty)$ for all $x \in X$. Let $g: K \rightarrow K$ and $f: K \times K \rightarrow Y$ with $g(x)=\left(x_{2}, x_{1}\right)$ and $f(x, y)=\left(x_{2}-y_{1}, x_{1}-y_{2}\right)$, where $x=\left(x_{1}, x_{2}\right)$ and $y=\left(y_{1}, y_{2}\right)$. It is easy to see that $f(g(x), y)=\left(x_{1}-y_{1}, x_{2}-y_{2}\right)$ for all $x=\left(x_{1}, x_{2}\right), y=\left(y_{1}, y_{2}\right) \in K$, and assumptions (i)-(iv) hold. If we set $D=K \backslash\{[0,1 / 2) \times[0,1 / 2)\}$ and $y_{0}=(1,1)$, then for all $x=\left(x_{1}, x_{2}\right) \in K \backslash D=[0,1 / 2) \times[0,1 / 2)$,

$$
f\left(g(x), y_{0}\right)=\left(x_{1}-1, x_{2}-1\right) \in(-\infty, 0) \times(-\infty, 0),
$$

that is, assumption (v) also holds. By Lemma 2.1 (iii), we have

$$
\begin{aligned}
\varphi(x) & =\left\{x^{*}=\left(x_{1}^{*}, x_{2}^{*}\right) \in K: f\left(g\left(x^{*}\right), x\right)=\left(x_{1}^{*}-x_{1}, x_{2}^{*}-x_{2}\right) \notin(-\infty, 0) \times(-\infty, 0)\right\} \\
& =K \backslash\left\{\left[0, x_{1}\right) \times\left[0, x_{2}\right)\right\}, \quad \forall x=\left(x_{1}, x_{2}\right) \in K .
\end{aligned}
$$

Obviously, for any given $x=\left(x_{1}, x_{2}\right) \in K, \varphi(x)$ is closed, and

$$
\bigcap_{x \in K} \overline{\varphi(x)}=\bigcap_{x \in K} \varphi(x)=\left\{\left[1, z_{2}\right]: z_{2} \in[0,1]\right\} \cup\left\{\left[z_{1}, 1\right]: z_{1} \in[0,1]\right\} \neq \emptyset .
$$


Thus, $S(f, g, K) \neq \emptyset$. Furthermore,

$$
S(f, g, K)=\bigcap_{x \in K} \overline{\varphi(x)}=\left\{\left[1, z_{2}\right]: z_{2} \in[0,1]\right\} \cup\left\{\left[z_{1}, 1\right]: z_{1} \in[0,1]\right\}
$$

is closed and compact.

REMARK 3.3. If $g$ is the identity mapping, then using Corollary 3.1, Theorem 3.1 gives existence of solutions for vector equilibrium problem based on the nonlinear scalarisation function.

If $g$ is the identity mapping and $X=Y$ in Theorem 3.1, then we obtain an existence Theorem for non-dominated vectorequilibrium problem.

Corollary 3.2. Let $X$ be a real Hausdorff topological vector space, $K$ a nonempty closed convex subset of $X$. Let $C: X \rightarrow 2^{X}$ be a point-to-set mapping such that for any $x \in X, C(x)$ is a proper, pointed, closed and convex cone in $X$. Suppose that $C=\bigcap_{x \in X} C(x)$ with int $\bar{C} \neq \emptyset$. Let the following assumptions hold:

(i) $f(x, x) \notin-\operatorname{int} C(x), \quad \forall x \in K$;

(ii) $y \mapsto f(\cdot, y)$ is affine;

(iii) $x \mapsto f(x, \cdot)$ is continuous;

(iv) the point-to-set mapping $W: K \rightarrow 2^{Y}$ has closed graph in $K \times Y$, where $W(x)=Y \backslash(-\operatorname{int} C(x)), \forall x \in K$;

(v) there exist a compact subset $D \subset K$, such that $\exists y_{0} \in K, \forall x \in K \backslash D$ such that

$$
f\left(x, y_{0}\right) \in-\operatorname{int} C(x)
$$

Then

$$
\bigcap_{x \in K} \overline{\varphi(x)} \neq \emptyset
$$

that is, $W(f, K) \neq \emptyset$, where

$$
\varphi(x)=\left\{x^{*} \in K: \xi_{k^{0}}\left(x^{*}, f\left(x^{*}, x\right)\right) \geqslant 0, \forall k^{0} \in \operatorname{int} \bar{C}\right\} .
$$

Furthermore, $W(f, K)$ is compact.

\section{REFERENCES}

[1] Q.H. Ansari, S. Schaible and J.C. Yao, 'The system of generalized vector equilibrium problems with applications', J. Global Optim. 22 (2002), 3-16.

[2] Q.H. Ansari and J.C. Yao, 'An existence result for generalized vector equilibrium problem', Appl. Math. Lett. 12 (1999), 53-56.

[3] A. Behera and L. Nayak, 'On nonlinear variational-type inequality problem', Indian $J$. Pure Appl. Math. 30 (1999), 911-923. 
[4] M. Bianchi, N. Hadjisavvas and S. Schaible, 'Vector equilibrium problems with generalized monotone bifunctions', J. Optim. Theory Appl. 92 (1997), 527-542.

[5] M. Bianchi and S. Schaible, 'Generalized monotone bifunctions and equilibrium problems', J. Optim. Theory Appl. 90 (1996), 31-42.

[6] E. Blum and W. Oettli, 'From optimization and variational inequalities to equilibrium problems', The Math. Student 63 (1994), 123-145.

[7] G.Y. Chen and G.M. Chen, Vector variational inequality and vector optimization, Lecture Notes in Econ. and Math. Systems 285 (Springer-Verlag, Berlin, Heidelberg, New York, 1987), pp. $408-416$.

[8] G.Y. Chen and B.D. Craven, 'Approximate dual and approximate vector variational inequality for multiobjective optimization', J. Austral. Math. Soc. Ser. A 47 (1989), 418-423.

[9] G.Y. Chen and S.H. Hou, 'Existence of solution for vector variational inequalities', in Vector Variational Inequalities and Vector Equilibria, (F. Giannessi, Editor), Nonconvex Optim. Appl. 38 (Kluwer Academic Publishers, Dordrecht, 2000), pp. 73-86.

[10] G.Y. Chen and Y.Q. Yang, 'Characterizations of variable domination structures via nonlinear scalarization', J. Optim. Theory Appl. 112 (2002), 97-110.

[11] K. Fan, 'A generalization of Tychonoff's fixed point theorem', Math. Ann. 142 (1961), 305-310.

[12] C. Gerth and P. Weidner, 'Nonconvex separation theorems and some applications in vector optimization', J. Optim. Theory Appl. 67 (1990), 297-320.

[13] F. Giannessi (Editor), Vector variational inequalities and vector equilibria, Nonconvex Optimization and its Applications 38 (Kluwer Academic Publishers, Dordrecht, 2000).

[14] C.J. Goh and Y.Q. Yang, 'Scalarization methods for vector variational inequality', in Vector Variational Inequalities and Vector Equilibria, (F. Giannessi, Editor), Nonconvex Optim. Appl. 38 (Kluwer Academic Publishers, Dordrecht, 2000), pp. 217-232.

[15] X.H. Gong, 'Efficiency and Henig efficiency for vector equilibrium problems', J. Optim. Theory Appl. 108 (2001), 139-154.

[16] A. Göpfert, H. Riahi, Chr. Tammer and C. Zalinescu, Variational methods in partially ordered spaces (Springer-Verlag, New York, 2003).

[17] N. Hadjisavvas and S. Schaible, 'From scalar to vector equilibrium problems in the quasimonotone case', J. Optim. Theory Appl. 96 (1998), 297-309.

[18] N.J. Huang, J. Li and H.B. Thompson, 'Implicit vector equilibrium problems with applications Math. Comput. Modelling 37(2003), 1343-1356'.

[19] I. V. Konnov and S. Schaible, Duality for equilibrium problems under generalized monotonicity, J. Optim. Theory Appl. 104 (2000), 395-408.

[20] I.V. Konnov and J.C. Yao, 'Existence of solutions for generalized vector equilibrium problems', J. Math. Anal. Appl. 233 (1999), 328-335.

[21] J. Li, N.J. Huang and J.K. Kim, 'On implicit vector equilibrium problems', J. Math. Anal. Appl. 283 (2003), 501-512.

[22] A.H. Siddiqi, Q.H. Ansari and A. Khaliq, 'On vector variational inequalities', J. Optim. Theory Appl. 84 (1995), 171-180.

[23] Y.N. Wu, 'Existence and convergence of solutions for vector equilibrium problems', Adv. Nonlinear Var. Inequal. 6 (2003), 49-58. 
School of Mathematics and Information China West Normal University

Nanchong

Sichuan 637002

Peoples Republic of China
Department of Mathematics Sichuan University

Chengdu

Sichuan 610064

Peoples Republic of China

e-mail: nanjinghuang@hotmail.com

njhuang@scu.edu.cn 